\title{
Minimally Invasive Management of Biliary Tract Injury Following Percutaneous Nephrolithotomy
}

\author{
Ata A Rahnemai-Azar ${ }^{1}$; Amir A Rahnemaiazar ${ }^{1}$; Rozhin Naghshizadian ${ }^{1}$; Jacob H Cohen ${ }^{2}$; \\ Iman Naghshizadian ${ }^{1}$; Brian F Gilchrist ${ }^{1}$; Daniel T Farkas ${ }^{1, *}$ \\ ${ }^{1}$ Department of Surgery, Bronx-Lebanon Hospital Center, Albert Einstein College of Medicine, New York, USA \\ ${ }^{2}$ Department of Urology, Bronx-Lebanon Hospital Center, Albert Einstein College of Medicine, New York, USA \\ ${ }^{*}$ Corresponding author: Daniel T Farkas, Department of Surgery, Bronx-Lebanon Hospital Center, Albert Einstein College of Medicine, Bronx, New York, USA. Tel: +1-7189601243, Fax: \\ +1-7189601370, E-mail:dfarkas@bronxleb.org
}

Received: May 6, 2014; Revised: June 20,2014; Accepted: June 26, 2014

\begin{abstract}
Introduction: Percutaneous nephrolithotomy is generally considered a safe option for the management of large complex or infectious upper urinary tract calculi. Biliary tract injury is a rare and potentially serious complication of percutaneous nephrolithotomy that can even lead to mortality, especially in cases where biliary peritonitis develops. All reported cases of biliary tract injury have been managed by either open or laparoscopic cholecystectomy.

Case Presentation: Herein for the first time, we report a 39-year old woman with biliary tract injury following percutaneous nephrolithotomy who was managed less invasively by insertion of a percutaneous cholecystostomy tube. The patient was discharged home shortly thereafter, and the tube was later removed at a follow up visit after a normal cholangiogram.

Conclusions: Biliary tract injury is a rare and potentially serious complication of percutaneous nephrolithotomy that can even lead to mortality. If a biliary tract injury is suspected during percutaneous renal procedures, diverting the bile away from the leak may resolve the problem without the need for a cholecystectomy. Ideally this can be done with ERCP and a stent, but in cases where this is not technically feasible; a percutaneous cholecystostomy can be successful at accomplishing the same result.
\end{abstract}

Keywords:Biliary Tract; Cholecystostomy Tube; Injury; Minimally Invasive; Percutaneous Nephrolithotomy

\section{Introduction}

After being first reported by Goodwin et al. percutaneous renal access has become an established procedure during the past few decades (1). Based on a review of the literature, injury to the biliary tract rarely occurs after percutaneous renal procedures but can lead to serious outcomes including death. Early recognition and intervention is critical to minimize the morbidity and mortality associated with this type of biliary tract injury. The evolution of peritonitis, because of delayed intervention, will make laparotomy inevitable and can potentially be associated with serious complications. In review of the few reported cases, all of these patients with a biliary tract injury underwent a cholecystectomy via either a laparoscopic $(2,3)$ or open procedure $(4-6)$. Herein we report the first case of biliary tract injury following a renal-targeted percutaneous procedure managed minimally invasively by insertion of a percutaneous cholecystostomy tube.

\section{Case Presentation}

A 39-year old woman, with past medical history of gastric bypass, was scheduled to undergo percutaneous nephrolithotomy (PCNL) for a $4 \mathrm{~cm}$ right renal pelvic calculus with partial staghorn extension to lower pole. At the time of passage of an 18-gauge needle to the right kidney under fluoroscopy guidance, bile stained fluid was aspirated and the procedure aborted. A large caliber ureteral stent and Foley catheter were placed to maximize urinary drainage and the patient admitted for observation. Overnight, the patient developed abdominal pain and became tachycardic and tachypenic. Laboratory studies showed an increased white cell count (19.9 $\times 10^{3}$ per microliter), while liver function tests stayed in normal range with total and direct bilirubin $0.6 \mathrm{mg} / \mathrm{dL}$ (0.2-1.3) and $0.2 \mathrm{mg} / \mathrm{dL}$ (0-0.4) respectively. Subsequently abdominal CT scan was performed which showed a large fluid collection in the right peri-hepatic and peri-nephric area (Figure 1). Interventional radiology was consulted to place a sub-hepatic drain.

Despite drainage of about 1 liter of bile stained fluid over the next 24 hours, the patient remained tachycardic and tachypenic with complaints of severe abdominal pain. General surgery was consulted and placement of a percutaneous cholecystostomy tube was recommended. Following this, the drainage from the sub-hepatic drain stopped and her clinical status improved significantly. Follow up ultrasonography revealed decreased peri-he- 
patic collection and the sub-hepatic drain was removed after three days. The patient was then discharged home in stable condition with the cholecystostomy tube. On post procedure day 17 , a tube cholangiogram revealed good flow of contrast into the duodenum without any evidence of leak or filling defect. Subsequently the tube was clamped and later removed in general surgery clinic. The kidney stone was later treated with staged ureteroscopy. At 6 months follow up, the patient is doing well without any complications and is stone free.

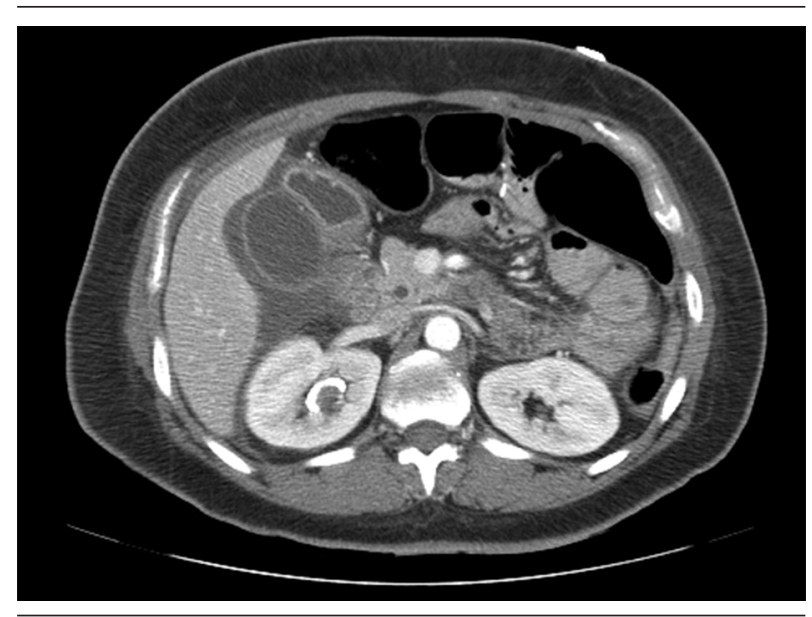

Figure 1. Abdominal CT Scan After Attempted Percutaneous Nephrolithotomy Showing Large Amount of Fluid Collection in Peri-Hepatic and Peri-Nephric Area

\section{Discussion}

Percutaneous nephrolithotomy has comparable results to open surgery with the advantage of being less invasive and having a shorter hospital stay (7). Although PCNL is generally considered a safe procedure, it can still be associated with serious and potentially life threatening complications. These include urinary tract infection, bleeding and damage to surrounding structures like colonic perforation, duodenal injury and pleural injury $(8,9)$.

Injury to the biliary tract during percutaneous renal access is a rare complication. In a study of 8000 ultrasound-guided punctures, only 4 incidents were reported and none of them was with a renal puncture target (10). In review of the literature, there are few cases of biliary tract injury during isolated nephrostomy (6) or percutaneous nephrolithotomy $(2,4,5)$, all of which have been managed by cholecystectomy.

Kontothanassis et al. (6) reported a case of gall bladder injury during an ultrasonography-guided nephrostomy done for urinary tract obstruction. Greenish fluid was noted during the procedure and the patient developed signs of abdominal distension and tenderness over the following 12 hours. The patient underwent a laparotomy and a cholecystectomy was performed.

Fisher et al. (2) reported another case of biliary tract injury after percutaneous access during PCNL. The patient developed abdominal discomfort and signs of peritoneal irritation on physical exam while a CT scan revealed pericholecystic and sub-hepatic fluid collection. Laparoscopic cholecystectomy was performed. Although gallbladder perforation secondary to percutaneous liver biopsy has been reported to be managed by insertion of percutaneous cholecystostomy tube, to our knowledge all reported cases of biliary injury during percutaneous renal accesses have been managed with cholecystectomy.

While bile peritonitis after PCNL is rare, it should always be kept in mind. Injury to the biliary tract should be suspected in patients who develop abdominal pain, peritoneal signs or any component of systemic inflammatory response syndrome (SIRS) post operatively. Aspiration of bile during procedure makes the diagnosis more likely. In our study as well as some previous case reports (6) the presence of "greenish fluid" was a clue to the biliary tract injury, although this finding does not always exist (2-4) and careful follow up is necessary after every percutaneous procedure. Certainly, prompt recognition of biliary tract puncture prior to renal access tract dilation is crucial to prevent further biliary tract damage.

Although some articles advise immediate cholecystectomy in gallbladder injuries during percutaneous nephrostomy (2), detection and appropriate drainage in a timely manner may avoid the need for cholecystectomy. One should consider this option even in the absence of peritonitis, if considerable amount of bile-colored fluid was initially aspirated during the procedure. Performing endoscopic retrograde cholangio-pancreatography (ERCP) and stent placement across the Sphincter of Oddi is a well-known and standard treatment approach for small bile leaks, such as cystic duct stump leaks. This allows bile to preferentially drain to the duodenum, so that the leak can heal. In this case with a history of gastric bypass, the option of performing an ERCP was significantly more complicated. As such, the decision was made to place a percutaneous cholecystectomy tube, with the same goal of allowing for preferential drainage of bile through the cholecystectomy tube rather than the area of leak. The patient's symptoms resolved almost immediately, and the leak was resolved without ever needing a cholecystectomy. The ease of management and the lower risk of peri-operative complications with better outcome make cholecystectomy a preferential option over laparotomy and cholecystectomy. If a biliary tract injury is suspected during percutaneous renal procedures, diverting the bile away from the leak may resolve the problem without the need for a cholecystectomy. Ideally this can be done with ERCP and a stent, but in cases where this is not technically feasible; a percutaneous cholecystostomy can be successful at accomplishing the same result.

\section{Authors' Contributions}

Amir A Rahnemaiazar: Substantial contributions to conception and design, Review article, drafting of the article, 
revising the article for important intellectual content. Rozhin Naghshizadian: Review article, drafting the article. Jacob H Cohen: Drafting article, revising the article for important intellectual content. Iman Naghshizadian: Review article, drafting the article. Brian F Gilchrist: Substantial contributions to conception and design, revising the article for important intellectual content. Daniel Farkas: Substantial contributions to conception and design, drafting of the article, revising the article for important intellectual content, final approval of the version to be published.

\section{Financial Disclosure}

None of the contributing authors have any conflict of interest, including specific financial interests or relationships and affiliations relevant to the subject matter or materials discussed in the manuscript.

\section{Funding/Support}

The entire publication fee will be sponsored by department of surgery, Bronx-Lebanon Hospital, Albert Einstein College of Medicine.

\section{References}

1. Goodwin WE, Casey WC, Woolf W. Percutaneous trocar (needle) nephrostomy in hydronephrosis. J Am Med Assoc. 1955;157(11):891-4.

2. Fisher MB, Bianco FJ Jr, Carlin AM, Triest JA. Biliary peritonitis complicating percutaneous nephrolithomy requiring laparoscopic cholecystectomy. J Urol. 2004;171(2 Pt 1):791-2.

3. Patel SR, Nakada SY. Biliary peritonitis after percutaneous nephrolithotomy: case studies and management concerns.J Endourol. 2010;24(11):1729-31.

4. Saxby MF. Biliary peritonitis following percutaneous nephrolithotomy. BrJUrol.1996;77(3):465-6.

5. Ricciardi S, Sallustio P, Troisi R. Life-threatening biliary complications after percutaneous nephro-lithotomy: a case report. Acta Chir Belg. 2007;107(3):336-7.

6. Kontothanassis D, Bissas A. Biliary peritonitis complicating percutaneous nephrostomy. Int Urol Nephrol.1997;29(5):529-31.

7. Preminger GM, Clayman RV, Hardeman SW, Franklin J, Curry T, Peters PC. Percutaneous nephrostolithotomy vs open surgery for renal calculi. A comparative study. JAMA. 1985;254(8):1054-8.

8. Wu P, Wang L, Wang K. Supine versus prone position in percutaneous nephrolithotomy for kidney calculi: a meta-analysis. Int Urol Nephrol. 2011;43(1):67-77.

9. Kumar A, Banerjee GK, Tewari A, Srivastava A. Isolated duodenal injury during relook percutaneous nephrolithotomy. Br J Urol. 1994;74(3):382-3.

10. Nolsoe C, Nielsen L, Torp-Pedersen S, Holm HH. Major complications and deaths due to interventional ultrasonography: a review of 8000 cases. JClin Ultrasound. 1990;18(3):179-84. 